\title{
The Role of TOF-SIMS in Nanomedicine
}

Beat A. Keller, Karl Mayerhofer ${ }^{1}$ (DOI 10.3884/0002.2.8)

\begin{abstract}
The toolbox of analytical techniques available today for materials scientists offers a wide selection of instruments for nearly every problem and application. Here we present a brief introduction into tissue imaging by time-of-fight secondary ion mass spectrometry (TOF-SIMS) for the non-expert. Possibilities and limitations of TOF-SIMS for identifying chemical components in biological matter are discussed. The potential of the technique is shown in a case study of $2 \mathrm{D}$ imaging of co-polymer based nanosized drug delivery containers embedded in mouse liver. Keller, B Nanomedicine 2009, 2.2:37-43
\end{abstract}

Keywords: TOF-SIMS, Cluster Primary Ion Beam Sources, Imaging in Cell and Tissue Research

\section{Introduction TOF-SIMS}

Time-of-flight secondary ion mass spectrometry (TOF-SIMS) is a very versatile surface sensitive analytical technique that yields elemental as well as molecular information from solid substrates ranging from biological materials to semiconductor applications. After the bombardment of a surface with energetic charged projectiles, the emitted secondary species originating from the topmost layers of the material are collected and analyzed with high mass resolution. The parallel detection scheme not only provides spectral information with high accuracy, but also allows the operation of the instrument in a microprobe mode to obtain chemically resolved images of the surface. The primary ion beam is scanned over a preselected surface area, and complete mass spectra are recorded from a pixel map. 3D distributions of chemical species are produced by alternating cycles of material ablation and image recording. In this dual beam mode, a sputter gun is removing a well defined layer of the material surface under accurately controlled lowenergy conditions. Afterwards a second ion beam is scanned over the crater area to specify the chemical composition of the freshly exposed surface. It is important to be aware that TOF-SIMS is generally a destructive analytical technique, and the quality of data is very sensitive to the chemical nature of sputter ions. High density data storage of mass and location information offers the possibility of retrospective analysis of samples containing previously unknown chemical species. In a reverse mode, the software can reconstruct the distribution of a chemical com- pound in a user-defined area.

TOF-SIMS applications of biomaterial surfaces have become a traditional field of the technique. Due to the important role of medical devices for modern medical treatment, and the increasing number of applications in all fields of trauma and reconstruction chirurgy the development and quality control of surfaces in medical systems has become vital for the progress of materials design. It is not within the scope of this paper to discuss TOFSIMS analysis of biomaterials. For a detailed discussion, the reader is referred to recent reviews and articles of the field [ 1 $-4]$, and the literature cited therein.

\section{Limitations of TOF-SIMS}

The pivotal idea of surface analysis is to answer basic questions about what is on the surface, how much, where. While TOF-SIMS provides detailed information about the nature and location of chemical compounds, it is less suitable to obtain quantitative data. The reason for this limitation is a strong dependence of secondary particle emission from the chemical environment, i.e. the matrix. The conductivity of the material influences the ability to neutralize the surface charge induced by the impact of charged primary projectiles. Although it is possible to compensate this effect to a certain degree, surface charging tends to degrade mass and spectral resolution due to instabilities of the acceptance angle of the mass analyzer. Even under stable emission conditions, the true chemical nature of surfaces can be masked by contaminations from sample preparation or improper handling. The preparation of complex materials as encountered in biological samples therefore requires careful preparation protocols to avoid the build-up of artifacts. If molecules are present in serum level concentrations (i.e. metabolites), secondary particles can only be detected using state-of-the-art cluster primary ion beam sources. Molecular fragments with a mass exceeding $\mathrm{m} / \mathrm{z}=1500$ are often formed in extremely low abundance and therefore not detected. Sophisticated principal component analysis (PCA) is applied to the data sets to identify unequivocally such signals $[5,6]$.

\section{TOF-SIMS with Cluster Primary Ion} Beam Sources

The desorption of secondary ions after the impact of energetic ions with typically several thousand electron volts was first used to by Benninghoven et al. [7] in 1976 to specify surface located organic molecules. However, it became soon evident that above the so-called static SIMS limit (ion dose about $10^{12}$ ions $/ \mathrm{cm}^{2}$ ) where more than about $1 \%$ of surface located molecules are consumed during measurement, an increasing amount of unspecific lowmass carbon builds-up through surface damage, causing initially compound-specific secondary signals to disappear. This later effect restricts the concentration of surface species that can be detected in static SIMS mode using monoatomic primary ions. Since the useful lateral resolution and the efficiency are connected, a

Swiss Federal Laboratories for

Materials Testing and Research

Ueberlandstrasse 129,

$\mathrm{CH}-8600$ Dübendorf, Switzerland 


\section{Analytical Nanomethods}

lower ion yield also affects the area from which an analytically useful information can be obtained. Therefore, it became apparent that the overall secondary ion yield must be increased in order to be able to detect low abundance chemical species on a surface. Experiments undertaken in the late $1980^{\prime}$ s using $\mathrm{SF}_{6}{ }^{+}$and $\mathrm{Cs}_{\mathrm{x}} \mathrm{I}^{+}$as primary ion sources $[8,9]$ showed improved signal intensities in polymers and organic thin films [10]. Other molecular ion species proved to be less effective and it was more difficult to maintain steady and uniform beam conditions. However, $\mathrm{SF}_{6}{ }^{+}$sources, still difficult to operate and providing only moderate beam current, could be commercialized by several manufacturers. The later development of $\mathrm{Au}_{n}{ }^{+}$, $\mathrm{Bi}_{n}{ }^{+}$and $\mathrm{C}_{60}{ }^{+}$guns overcame these limitations $[11,12]$ and are state-of-the-art today. Recent calculations indicate that the damage accumulation rate is in many cases lowest for fullerene primary ions, probably due to the low kinetic energy of individual carbon atoms as compared to the atoms in a small polyatomic cluster [13]. Cluster primary ion sources produce an up to 1000 times higher amount of secondary ions and due to the less vigorously fragmentation also allow for the detection of very specific high-mass molecular fragments, typically encountered in biological systems. For a recent review on cluster primary ion sources, the reader is referred to [14].

The most striking advantage ofTOF-SIMS using cluster ion sources, however, is the fact that the high intensity of signal arising from individual pixels makes it possible to detect small amounts of a species from a small area, i.e. obtaining a smaller useful lateral resolution as required in many biological applications. As the pixel dimension (typically $256 \times 256$ pixel per area) approaches sub-micrometer size, the number of analytically useful surface molecules located within this area is in the micromole range.

\section{Applications of TOF-SIMS Imaging in Cell and Tissue Research}

Sample preparation and handling are significant for the quality of TOF-SIMS data obtained from biological matter. In a recent paper, Kurczy et al. [15] concluded that both, the nature of the projectile and sample preparation protocols are equally important to acquiring successful information from biological samples.

Preparation of Biological Matter for TOFSIMS measurements
Sample preparation for imaging TOFSIMS experiments include several important issues that each influences the quality of the acquired data. Most notable these are:

\section{A. specimen collection}

B. specimen handling (freezing, embedding, chemical fixation, etc).

C. sectioning and mounting manipulations

\section{D. measurement temperature}

The collecting of biological matter, which is either harvested from an organ (liver, kidney, spleen etc.), originating from a biopsy, or taken from a cell culture is the very first step of a successful bio imaging experiment. Several instruments like biopsy needles or collecting plates are used for this purpose. Before transferring the tissue sample to a suitable substrate holder, it is imperative to freeze the specimen as fast as possible either in liquid pentane, isopentane or another appropriate water free cooling medium.

As is well documented, freezing generally damages cell membranes, and therefore reduces the histological information from biological samples. This effect is most prominent after slow freezing and in large volume samples. The former process promotes ice crystal formation, and the latter reduces the cooling rate due to the Leidenfrost effect. Large thermal gradients inside the specimen would be the consequence. Since the rate of freezing is more important than the actual end-temperature, it is important to avoid such behavior. Shock-freezing in hydrocarbon solvents kept at liquid nitrogen temperature has been established as working protocol to prepare biological samples at freezing temperature. However, even if a specimen is quickly enough frozen down to a vitreous (amorphous) state, it may not stay that way for a long time because vitreous ice forms an unstable state. Above $-212{ }^{\circ} \mathrm{C}$, amorphous ice begins to gradually undergo a phase restructuring process to cubic ice, associated with volume expansion. As a consequence the water stretches and starts to penetrate cell membranes. While this is a slow process, it still sets rather tight time limits to the measurement, and particularly at the time of sample introduction. Modern TOF-SIMS equipment is outfitted with liquid nitrogen cold traps to assist cold temperature measurements, but the experiment still requires experience and skills. The correct way to prepare samples of biological origin for TOF-SIMS imaging investigations has stimulated a long debate among specialists. For a recent review of tissue preparation for TOF-SIMS measurements, the reader is referred to [16] and the literature cited therein.

For some time, chemical fixation protocols, using glutaraldehyde or a water/ acetic acid mixture have been used to prepare samples. Glutaraldehyde reacts through its two aldehyde groups with one primary amine group of a protein. Thus, glutaraldehyde acts as protein linker, and preserves cell structure, although in a completely water-free state. On the other hand, treatment with a water/acetic acid mixture dissolves the cell membrane. The remaining tissue sample mainly consist of the nucleus (or parts of it), and the cytoskeleton of cells. Certainly, one does not expect to find the original native state of tissue after such harsh chemical treatment. Nevertheless, chemical fixation protocols have been frequently used to prepare biological samples $[17,18]$.

Since a freshly cut sample is unlikely to be completely flat, one must also consider the roughness of the specimen on the molecular level. In order to reflect the real distribution of molecular species within a sample, it is important to consider this fact. In particular if a $3^{D}$ image is reconstructed from a depth profiling experiment, e.g. using $\mathrm{C}_{60}{ }^{+}$sputter ions. It is immediately evident that for example a spherical object can be distorted due to impact angle dependent shadowing effects during etching. A better way to handle this problem would be the construction of a slicing instrument that produces a series of freshly prepared surfaces from which 2D molecular images are measured in the static SIMS mode and subsequently stored. The 3D map is then reconstructed from slices rather than from a damage plagued sputter profile.

In recent years, SIMS bioimaging has been applied to a range of tissue specimen using static TOF-SIMS. Among the most promising studies were the mapping of fatty acids, lipids, and vitamin E, among others $[12,19]$ in mouse and rat brain sections. Distribution maps showed the location of these rather large molecules in the intracellular medium by pooling relevant fragments. Molecular details of low molecular weight compounds located at the single cell level are obviously much more difficult to extract. First of all, the amount of available molecules is generally very 
small, and secondly, the focus requirements of the primary ion beam are very demanding. However, several studies have been performed, which reveal some details about the molecular organization of single cell samples [20].

\section{D Mapping of artificial Polymers in Tis- sue - a TOF-SIMS case Study}

The advantage of targeting specific cells and components of biological interest in tissue samples has been outlined by Broz et al. [21]. In particular the possibility to transport drugs and molecules with diagnostic functionality intact, i.e. by avoiding the harsh digestion process, to certain parts of a vertebrate body has been proposed as therapeutic concept with great potential. Conventional ways to deliver drugs for certain therapies are not very specific, and generally drug action can not be restricted to well defined parts of a body. Therefore the amount of drug or diagnostic material often exceeds the amount which is minimally necessary to perform the desired action. The consequences for the patient are oftentimes very uncomfortable and sometimes accompanied by sickness and the danger of allergic reactions. Although the concept of drug delivering by means of small containers which are specifically targeting the membrane proteins of a certain cell type is well documented, the interplay between recognition and controlled drug release by subsequent trigger action is not easy to combine. The experimental proof of this principle has yet to be shown.

A first important step towards this goal is the $2 \mathrm{D}$ mapping of drug delivery containers of nanometer dimensions. Here we present the application of time-of-flight secondary ion mass spectrometry (TOFSIMS) to produce chemically resolved surface images of thin mouse liver slices containing $50 \mathrm{~nm}$ sized drug delivery containers. Characteristic molecular fragments of PDMS (part B of an ABA triblock copolymer) can be identified in the TOFSIMS image and reflect the two-dimensional distribution of the containers on a scale of $500 \times 500 \mu \mathrm{m}^{2}$. The chemical composition of the polymer is shown in figure 1.

As shown in figure 1, the synthetic nanocontainersconsistofahydrophilic(PMOXA = poly(2-methyloxazoline) and a hydrophobic (PDMS = poly (dimethylsiloxane) building block, giving the container wall an amphiphilc character [21]. The ABA copolymer forms stable vesicles in aqueous media with a diameter of $50-100$ $\mathrm{nm}$ (in some cases also up to $250 \mathrm{~nm}$ ). The PMOXA-PDMS-PMOXA copolymer structure is biocompatible and shows very little susceptibility to non-specific plasma protein binding (e.g. serum albumin). Therefore the material has been chosen by Broz et al. [21, 22] as model for cell targeting using fluorescent markers for position location

While the use of confocal microscopy is a key technology in nanomedicine due to its sensitivity and ability to perform time-resolved measurements that allow the experimental investigation of interaction processes, the method requires tagging of molecules with fluorophores [23]. This principle is not always accessible in a straightforward way, and therefore applications are sometimes prone to synthetic restrictions or artifacts. Time-of-flight secondary ion ,mass spectrometry offers an alternative, label-free way to record spatial distributions of chemical species within samples of biological origin.

\section{Experimental Section}

TOF-SIMS spectra and 2D fragment distribution maps were acquired using a TOFSIMS $\mathrm{V}$ instrument (IonTof $\mathrm{GmbH}$, Münster, Germany) equipped with a $\mathrm{Bi}$ liquid metal primary ion source angled at $45^{\circ}$ to the sample plane. The Bi-cluster ion gun ( $25 \mathrm{keV}, 10 \mathrm{kHz}$ ) produces maximum pulsed target currents of $1 \mathrm{pA}$ for $\mathrm{Bi}^{+}, 0.45 \mathrm{pA}$ for $\mathrm{Bi}_{3}{ }^{+}$and $0.3 \mathrm{pA}$ for $\mathrm{Bi}_{3}{ }^{2+}$, respectively, in a high current bunched mode. However, the operation of the instrument in a mode for simultaneous high mass resolution in combination with high spatial resolution, as required for the detection of small molecules within a tissue sample, will require some alterations in the source settings. The trade-off for working with this option would be a lower target current and therefore also a longer measurement time for sufficient signalto-noise ratio. Alternatively, the instrument can be operated in a so-called burst alignment mode, whith a reasonable good lateral resolution and beam brightness, but only with unit mass resolution, which is only acceptable for high mass imaging with minimal mass interferences. An extraction lens biased at $\pm 2000 \mathrm{~V}$ collected positive and negative secondary ions which were subsequently analyzed in a reflectron type mass filter. A maximum mass resolution of $\mathrm{m} / \Delta \mathrm{m} \geq 10^{\prime} 000$ was achieved with this experimental set-up. A pre-biased microchannel plate detector operating in a single ion counting mode was used to obtain statistical numbers of secondary ion counts for each fragment. The sample itself was mounted onto a $\mathrm{LN}_{2}$-cooled standard stage obtained as accessory from lonTOF GmbH (Münster, Germany). A maximum temperature of $-120^{\circ} \mathrm{C}$ was maintained through all measurements.

TOF-SIMS images were recorded by rastering the primary ion beam across a preselected area of interest on the sample. As standard, a pixel resolution of $128 \times$ 128 or $256 \times 256$ was chosen. A full data set consisted of one complete mass spectrum for each pixel. However, very small features are difficult to analyze because in this case the size of the biological units approaches the minimum size of the pixel. For features smaller than this dimension, it becomes necessary to compare neighboring pixel intensities rather than averaging over the signal from a large number of pixels. The underlying concept of useful (or best) lateral resolution $(\Delta \mathrm{L})$, defined as the side length of the minimum square area from which a number of $\mathrm{N}$ secondary ions of a certain mass are desorbed and detected has been introduced by Benninghoven and Kötter [24]. Several authors defined a number $\mathrm{N}=4$ as adequate for most samples. One might, however speculate if this value is sufficiently describing the case of a small number of pixels. The influence of counting statistics in TOF-SIMS imaging experiments is still not fully explored.

Samples of rat kidney, liver and spleen

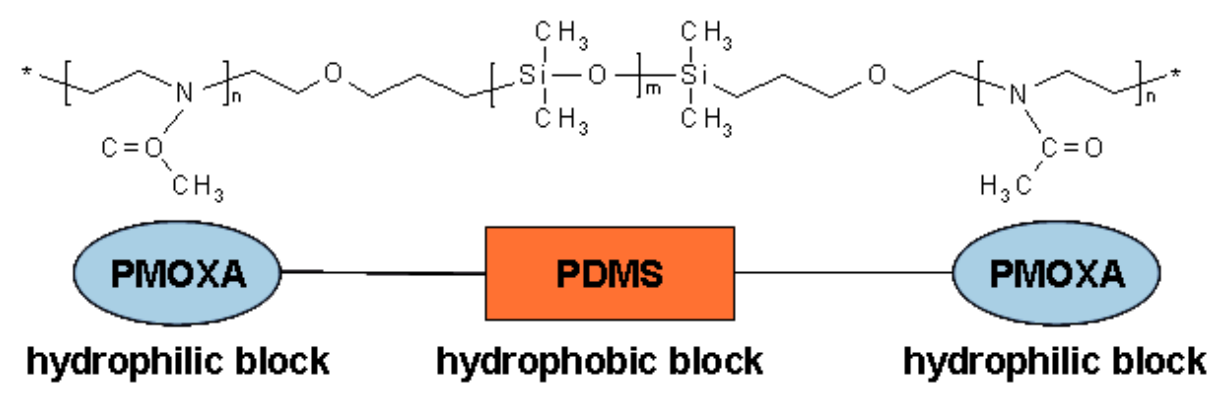

Figure 1: Chemical structure of drug delivery container wall polymer according to ref. [14] 


\section{Analytical Nanomethods}

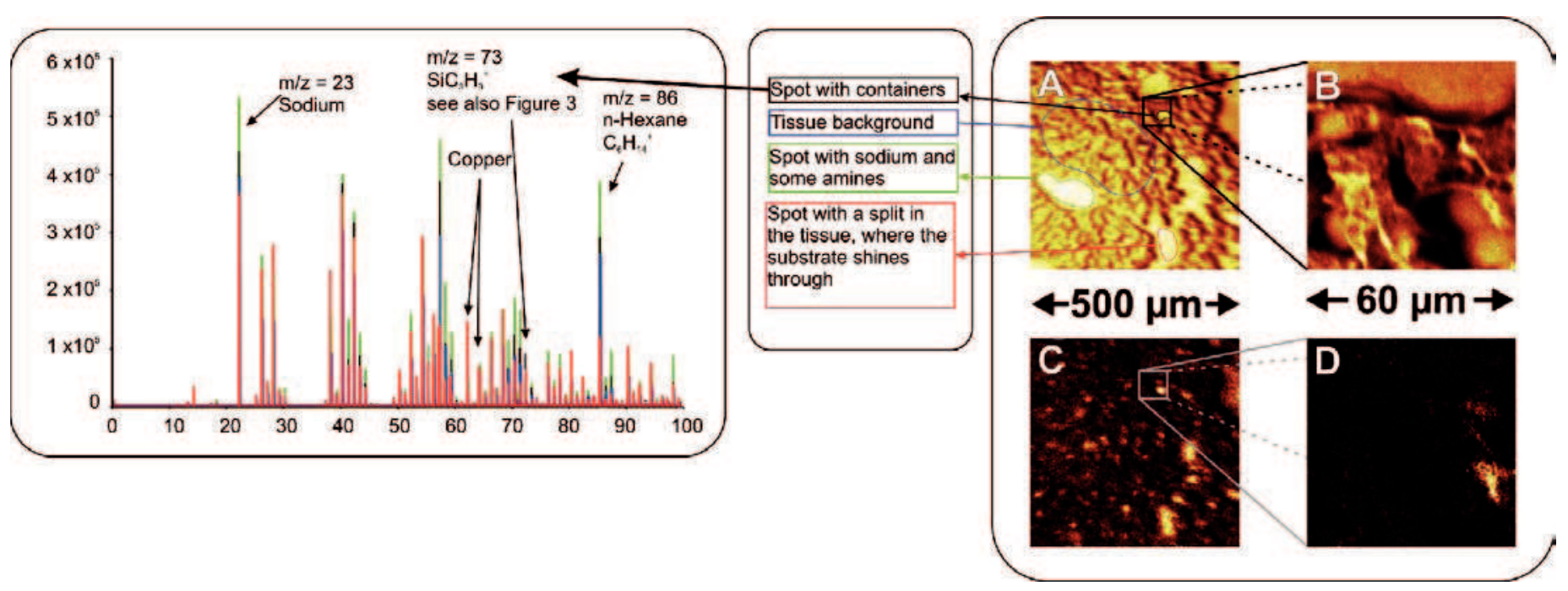

Figure 2: TOF-SIMS images of positively charged secondary ions obtained from mouse liver slices with A: total ion image, B: total ion image of rectangle in A, C: and D: distribution of mass $\mathrm{m} / \mathrm{z}=73$ (characteristic fragment of drug delivery container. Arrows indicate the area distribution of characteristic fragments found in the survey mass spectrum on the left side.

were prepared by cutting thin slices using a standard laboratory microtome, transferring the tissue sections onto gold coated steel plates and shock freezing the specimen by immersing the plates into liquid nitrogen. After this procedure, the samples were stored in a $\mathrm{LN}_{2}$-storage container and kept in a frozen state until measurement. The transfer of the tissue slices onto the TOF-SIMS cold stage was performed in a portable glove box tent kept under inert and water free conditions to avoid excessive condensation of water and hydrocarbons onto the substrate. In order to avoid secondary contaminations caused by laboratory equipment, all solvents, cutting blades, tweezers, beakers and $\mathrm{LN}_{2}$-Dewar containers were carefully cleaned before use. However, it was not possible to completely wipe-out all components of the microtome cutting instrument.

\section{TOF-SIMS Images of Mouse Liver Slices}

The TOF-SIMS spectrum of positively charged secondary ions in the so-called fingerprint region between masses $\mathrm{m} / \mathrm{z}=$ $1-100$ is shown on the left-hand side of figure 2 . Within one mass spectrum, there are a few hundred signals which in theory can each be assigned to a multitude of chemical compounds. Therefore, the identification of specific signals from the drug delivery containers can be difficult and time consuming. This rather complex situation is shown in figure 3 .

Therefore, the identification of small features within TOF-SIMS images requires a two-fold approach:
1. The possibility to perform TOF-SIMS imaging with high mass resolution and high lateral resolution at the same time. 2. A standardized approach to verify observed features using either mathematical tools or an alternative analytical technique that acquires images in another form than ion-matter interaction, e.g. fluorescence imaging $[23,25]$.

The development of primary ion beam technology meets the first requirement by operation the source in a so-called burst mode, where the ion pulse is separated into 4 sub-pulses (patented technology of IonTof $\mathrm{GmbH}$, Münster, Germany). In this mode of operation, it is possible to achieve an average mass resolution of about $\mathrm{m} / \Delta \mathrm{m}=3000$ for fragment signals in the range of $m / z=1-300$. Even with these restricted figures, the acquisition of secondary ion images is possible for specific masses. However, in some cases the unit resolution of other existing operation modes is sufficient to separate a specific fragment signal from interfering masses. Note that the relatively low mass resolution in our experiments is due to consid-

\begin{tabular}{|l|l|l|l|}
\hline Ion & Origin & Mass & $\mathrm{m} / \Delta \mathrm{m}$ \\
\hline $\mathrm{C}_{3} \mathrm{H}_{5} \mathrm{O}_{2}^{+}$ & tissue & 73.029 & 4058 \\
\hline $\mathrm{Si}\left(\mathrm{CH}_{3}\right)_{3}^{+}$ & container & 73.047 & - \\
\hline $\mathrm{H}_{9} \mathrm{O}_{4}^{+}$ & water & 73.050 & 24349 \\
\hline $\mathrm{C}_{3} \mathrm{H}_{7} \mathrm{NO}^{+}$ & Tissue (???) & 73.053 & 12174 \\
\hline $\mathrm{C}_{4} \mathrm{H}_{11} \mathrm{~N}^{+}$ & tissue & 73.089 & 1739 \\
\hline
\end{tabular}

Table 1: fragments around nominal mass $\mathrm{m} / \mathrm{z}=73$ which contribute to the signal interference at low mass resolution

erable charging of the sample rather than a limited instrumental mass resolution. In theory, fragments of water are not separable from PDMS fragments at mass 73 (see table 1). However, under realistic experimental conditions, the mass of PDMS fragments tends to shift for about +200 ppm, thus becoming distinguishable from ice fragments.

The definition of a measurement technique for data validation is in no way as easy as it seems. Only methods with comparable interaction and pixel definition as found in secondary ion mass spectrometry will yield data sets that allow for the confirmation of observed distribution of metabolites or other molecules of biological interest.

Positive-ion images of rat liver sections are shown in images $A$ to $D$ of figure $4 . A$ $\mathrm{Bi}^{+}$primary ion beam, producing a pulsed ion current of $2 \mathrm{pA}$ on the target was used in the burst mode operation mode. The distribution maps in figure 4 show 500 $x 500 \mu \mathrm{m}^{2}$ areas from left to right: $A=$ total ion image, $B=$ distribution of fragment mass $\mathrm{m} / \mathrm{z}=73, \mathrm{C}=$ sum of specific 


\section{Analytical Nanomethods}

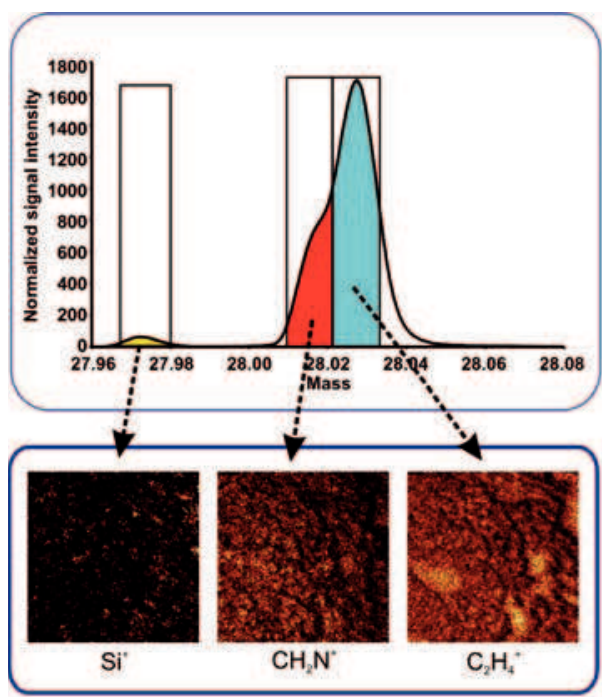

Signals at nominal mass 28

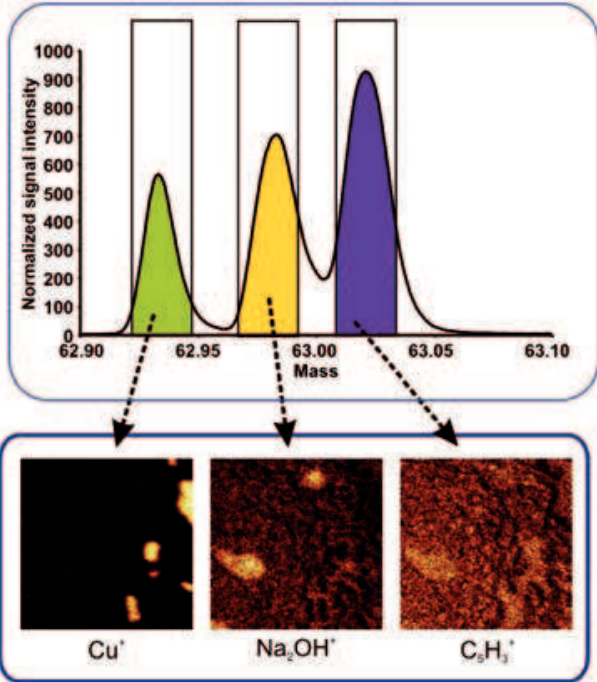

Signals at nominal mass 63

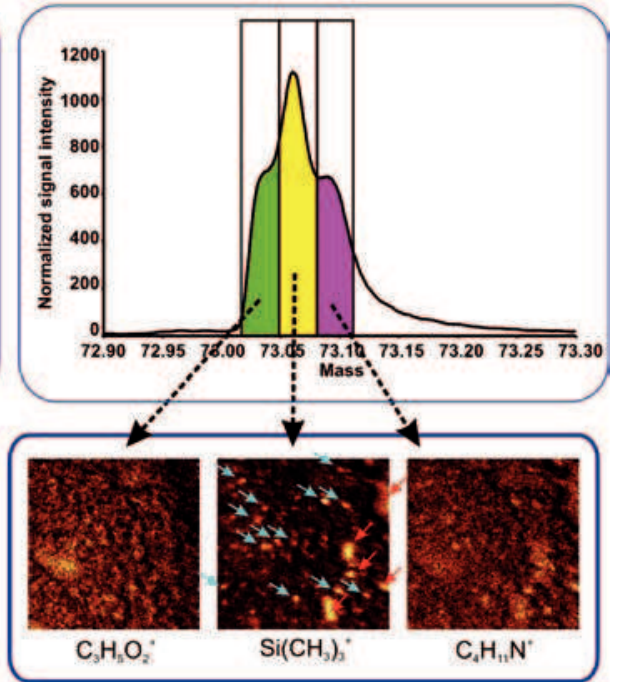

Signals at nominal mass 73

Figure 3: High resolution spectra and TOF-SIMS images $\left(500 \times 500 \mu \mathrm{m}^{2}\right)$ of mouse liver slices, showing mass interferences at specific fragment mass $(\mathrm{m} / \mathrm{z}=73)$ of drug delivery containers. The situation clearly shows the need for high mass resolution spectroscopy combined with high lateral resolution imaging.

mass fragments of lipids and $D=$ overlay image. The position of drug delivery containers are marked as red dots fin figure 4D. Domains of an unknown compound, characterized by a fragment with a specific mass of $\mathrm{m} / \mathrm{z}=269.27$ are shown as well as silicon contaminations arising from the underlying substrate which suggest the presence of small fractures and holes in the sample. Despite the fact that all measurements were performed at a temperature less than $-125^{\circ} \mathrm{C}$, tissue samples were gradually loosing water, probably subliming to the cold fingers of the $\mathrm{LN}_{2}$ cooling equipment. Although the integrity of the specimen may be altered somewhat by this effect, the positions of the containers are clearly visible as individual red points. The overlay image further indicates that no drug delivery containers were found within the lipid rich area. Whether this finding has some therapeutic value is not clear and the answer to it will need further investigations.

A minimum raster size of $60 \times 60 \mu \mathrm{m}^{2}$ was still yielding statistically useful data from our sample. However, as discussed before, the significance of low signal levels found in some of the pixels are not yet fully explored. Here, additional experiments providing refined statistical values have to be undertaken in the future.

\section{Conclusions}

Although ion-induced sputtering of organic material is a highly destructive process in general, a TOF-SIMS instrument can be operated at a minimal destructive mode while at the same time maintaining well controlled low-temperature $\left(-140^{\circ} \mathrm{C}\right)$ conditions over the measurement time. With this configuration, TOF-SIMS pro- vides an analytical technique for 2D-mapping of chemical species in tissue and cell samples. Here we present chemically resolved images of thin slices of mouse liver tissue, containing small ( $50 \mathrm{~nm}$ ) drug delivery containers. The container wall consists of a biocompatible co-polymer. The distribution of characteristic molecular fragments of PDMS (part B of an ABA triblock copolymer) can be identified in the TOF-SIMS image and the location of the containers on a scale of $500 \times 500 \mu^{2}$ can be specified. Presently the analysis area maintaining minimal material degradation is limited to $60 \times 60 \mu \mathrm{m}^{2}$. In the future we plan to downscale this value in order to obtain 2D-images of low-abundance metabolites.
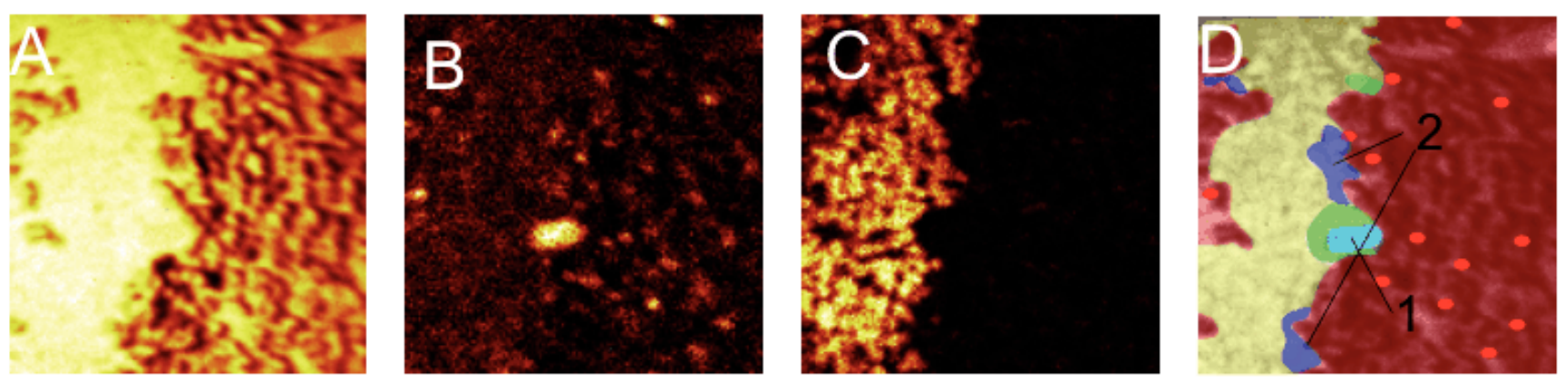

Figure 4: Positive-ion TOF-SIMS images $\left(500 \times 500 \mu \mathrm{m}^{2}\right)$ of mouse liver with A: total ion image, $B$ : distribution of fragment $\mathrm{m} / \mathrm{z}=73, \mathrm{C}:$ distribution of specific mass for lipids and D: overlay image showing drug delivery containers as red dots and the location of lipid rich areas as grey-green domains. Note that no containers are detected within the large lipid domain. Dark blue areas (2) indicate a yet unknown metabolite $(\mathrm{m} / \mathrm{z}=$ $269.27 a n d$ the light blue (1) spot is a hole in the slice with a silicon contamination from the substrate shining through. All measurements were performed at $\mathrm{T}<-125^{\circ} \mathrm{C}$. 


\section{Analytical Nanomethods}

\section{Acknowledgements}

The authors with to thank Prof. P. Hunziker and Mrs. R. Baenziger from University Hospital Basel for stimulating discussions, providing mouse liver, kidney and spleen samples and help to prepare the tissue slices.

\section{References:}

1. B. D. Ratner in R. L. Reis and S. Weiner, eds, NATO Science Series II: Mathematics, Physics and Chemistry, Kluver Academic Publishers, Netherlands, 2004, "Surface Analysis of Biomaterials and Biomineralization"

2.J. Grams, New Trends and Potentialities of ToF-SIMS in Surface Studies, "Biomaterials, Biomolecules, Biologic, Biomaterials, Biomolecules, Biological Systems and Applications in Medicine", Nova Science Publishers, Inc, Hauppauge, USA, 2007, $21-59$

3. Chilkoti, A. "Biomolecules on Surfaces", in TOF-SIMS: Surface Analysis by Mass Spectrometry, Vickerman, J. C. , Briggs, D., eds, IM Publishers, Chichester, U.K., 2001, p.627 - 650

4. Wagner, M. S., Castner, D. G., Applied Surface Science, 2004, 231 - 232, 366 -376, "Analysis of Adsorbed Protein Films by Static TOF-SIMS"

5. Wagner, M. S., Pasche, S., Castner, D. G., M. Textor, M., Analytical Chemistry, 2004, 76, 1483-1492, "Characterization of Poly(L-Lysine)-graft-Poly(Ethylene Glycol) Assembled Monolayers on Niobium Pentoxide Substrates using ToF-SIMS and Multivariate Analysis"

6. Graham, D. J., Wagner, M. S., Castner, D. G., Applied Surface Science, 2006, 252, 6860 - 6868 "Information from complexity: Challenges of TOF-SIMS data interpretation"

7. Benninghoven, A., Jaspers, D., Sichterman, W. Appl. Phys. 1976, 11, 35 - 39, "Secondary-Ion Emission of Amino Acids"

8.Blain, M. G., Della-Negra, S., Joret, H., Le Bayec, J., Schweikert, E.A. Phys Rev. Lett. 1989, 63, $1625-1628$, "Secondaryion yields from surfaces bombarded with keV molecular and cluster ions"

9. Appelhans, A. D., Delmore, J. E. Anal. Chem. 1989, 61, 1087 - 1093, "Comparison of Polyatomic and Atomic Primary Beams for Secondary Ion Mass Spectrometry of Organics"

10. Gillen, G., Roberson, S. Rapid communications in Mass Spectrometry 1998, 12, 1303-1312, "Preliminary evaluation of an $\mathrm{SF}_{5}^{+}$polyatomic primary ion beam for analysis of organic thin films by secondary ion mass spectrometry"
11. Touboul, D., Halgand, F., Brunelle, A., Kersting, R. et al. Anal. Chem. 2004, 76, 1550 - 1559, "Tissue molecular ion imaging by gold cluster ion bombardment" 12. Touboul, D., Kollmer, F., Niehuis, E., Brunelle, A., et al. J. Am. Soc. Mass Spectrom. 2005, 16, 1608 - 1618, "Improvement of biological time-of-flight secondary ion mass spectrometry imaging with a bismuth cluster ion source"

13.Postawa Z., Czerwinski, B., Szewczyk, M., Edward J. Smiley, E. J., Winograd, N., Garrison, B., J. Phys. Chem. B 2004, 108, 7831 - 7838, "Microscopic Insights into the Sputtering of $\mathrm{Ag}\{111\}$ Induced by $\mathrm{C}_{60}$ and Ga Bombardment"

14. Winograd, N. Analytical Chemistry 2005, 77, 143A - 149A, "The Magic of Cluster SIMS"

15.Kurczy, M. E., Piehowski, P.D., Parry, S. A., Jiang, M., Chen, G., Ewing, A. G., Winograd, N. Appl. Surf. Sci. 208, 255, 1298 - 1304, "Which is more important in bioimaging SIMS experiments - The sample preparation or the nature of the projectile?"

16. McDonnell, L. A. and Heeren, R. M. Mass Spectrometry Reviews 2007, 26, 606-643, "Imaging mass spectrometry" 17. Levi-Setti, R., Gavrilov, K. L., Strissel, P. L., Strick, R. Appl. Surf. Sci 2004, 231, 479 - 484, "Ion microprobe imaging of ${ }^{44}$ Ca-labeled mammalian chromosomes" 18. Levi-Setti, R., Gavrilov, K. L., Neilly, M. E., Appl. Surf. Sci. 2005, 252, 6765-6769, "Cations in mammalian cells and chromosomes: Sample preparation protocols affect elemental abundances by SIMS"

19. Sjovall, P., Lausmaa, J., Johannson, B. Analytical Chemistry 2004, 76, 4271-4278 "Mass Spectrometric Imaging of Lipids in Brain Tissue"

20. Roddy, T.P., Cannon, D.M., Ostrowski, S.G., Winograd, N., Ewing, A.G. Anal. Chem 2002, 74, 4020 - 4026, "Identification of Cellular Sections with Imaging Mass Spectrometry Following Freeze Fracture"

21. Broz, P., Benito, S. M., Saw, CL, Burger, P., Heiderd, H., Matthias Pfisterer, M., Marsch, S., Meier, W. Hunziker, P. Journal of Controlled Release 2005, 102, 475 - 488, "Cell targeting by a generic receptor-targeted polymer nanocontainer platform"

22. Broz, P., Driamov, S. ,Ziegler, J., BenHaim, N., Marsch, S., Meier, W., Hunziker, P. Nano Letters 2006, 6, 2349-2353, „Toward Intelligent Nanosize Bioreactors: A pH-Switchable, Channel-Equipped, Functional Polymer Nanocontainer"
23. Pickup, J., Khan, F., Zhi, Z.-L., Saxl, T. Europ. J. Nanomed. 2009, 2 , 16 - 21, "Fluorescence in Nanometrology: its Potential Role in Diabetes Research and Management"

24. Kötter, F., Benninghoven, A. Appl. Surf. Sci 1998, 133, 47 - 57, "Secondary ion emission from polymer surfaces under $\mathrm{Ar}+, \mathrm{Xe}+$ and $\mathrm{SF}_{5}+$ ion bombardment"

25. Valeur, B. "Molecular Fluorescence - Principle and Applications", Wiley-VCH (Weinheim), 2002 